\title{
Hinweise zur Qualitätssteigerung in der kommerziellen Politikberatung
}

\author{
Eckhard Jesse
}

Rezension von Shamim Rafat (2012). Ethik und Qualität in der Politikberatung. Zur Entwicklung von professionellen Standards und Grundsätzen. Bielefeld: Transcript.

Politikberatung ist in Deutschland lange vernachlässigt worden. Mittlerweile sieht das anders aus, sodass auch die einschlägige Literatur an Umfang zunimmt. Eine wichtige Konsequenz der Professionalisierung der Politikberatung muss lauten: Diese hat wissenschaftlichen Standards zu genügen. Das ist der Untersuchungsgegenstand dieser Studie, die sich auf die kommerzielle Politikberatung bezieht.

Die bei Karl Rudolf Korte angefertigte Dissertation, die auf einer früheren Arbeit basiert (Rafat (2006), Qualitätsmanagement in der Politikberatung. Professionalisierung der Politikberatung durch die Entwicklung von Qualitätskriterien? Münster: LIT), stellt im Untersuchungsrahmen die Bedeutung von Ethik und Qualität in der Politikberatung heraus. Dem Autor geht es um die Empirie, die Theorie sowie um die Praxisorientierung. Tatsächlich entfaltet der Autor unter „Theorie“ normative Grundzüge. Und die „Praxisorientierung“ ist teils empirisch, teils normativ ausgerichtet. Im zweiten Kapitel werden theoretische Grundlagen der Politikberatung gelegt. Ein Politikberater müsse qualifiziertes Fachwissen mitbringen und spezifische Qualifikationen besitzen (z.B. sprachliche Flexibilität). Die Auffassung Rafats, bisher habe sich eine allgemeingültige Definition des Begriffs „Politikberatung“ nicht durchsetzen können, dürfte auch nach seiner Arbeit weiter Bestand haben. Das vierte Kapitel berücksichtigt Formen, Berufspraxis und Entwicklungsprozesse der hiesigen Politikberatung. Die wissenschaftliche Politikberatung kommt ebenso zur Sprache wie das Lobbying und die kommerzielle Politikberatung, wobei Rafat zu Recht auf die fließenden Grenzen verweist. „Ohne ein wissenschaftlich begründetes Wissen ist in allen drei Bereichen der Politikberatung kein Staat zu machen" (S. 62). Im nächsten Kapitel wird die Zunahme der Professionalisierung der Politikberatung nachgezeichnet, danach das Forschungsdesign dieser Arbeit erläutert. Das sechste Kapitel schildert die Ergebnisse der OnlineBefragung unter Politikberatern. Moralische Standards spielten für das Selbstverständnis der Politikberater keine Rolle. Rafat sieht das als „defizitär“ (S. 150) an.

Der Autor stellt anschließend einen Kriterienkatalog auf, der moralisch-rechtliche, ethische und instrumentell-technische Standards einbezieht. Dieser Katalog soll „normativ gehaltvoll und empirisch anschlussfähig“ (S. 155) sein. Zu den ethischen Standards rechnet Rafat Unabhängigkeit, Neutrali- tät, Objektivität, Integrität und Loyalität, Vertraulichkeit, Wahrhaftigkeit, Transparenz. Allerdings wird hier nicht der Unterschied zwischen „Neutralität“ und „Objektivität“ deutlich. Bei den instrumentell-technischen Standards finden Aufnahme: fairer Wettbewerb; seriöse Preisbildung; seriöse Werbung; Vermeidung unvereinbarer Tätigkeiten (jeweils als Prämissen); poltische Expertise; kommunikative Kompetenz; Lösungskompetenz; strategisches Verständnis und Orientierung an Stakeholdern; Fortbildung und fachliche Weiterentwicklung (jeweils als fachliche Qualitätskriterien); konkrete Zielsetzung und Auftragsformulierung für den Beratungsprozess; klare Definition, Identifikation und Einsetzung von Ressourcen und Instrumenten; klare Kompetenzaufteilung der Verantwortlichkeiten und Zuständigkeiten mit dem Auftraggeber; sachgerechte Einsatzplanung; Auftragssteuerung im gesamten Beratungsprozess; Zuverlässigkeit der Leistung; kontinuierliche Beobachtung der Rahmenbedingungen im Beratungsprozess; kontinuierlicher Informationsaustausch mit dem Auftraggeber; systematische Dokumentation des Beratungsprozesses; transparentes Controlling der eigenen Dienstleistung (jeweils als handwerkliche Qualitätskriterien).

$\mathrm{Zu}$ Recht werden diese Standards anschließend einem Praxistest in Experteninterviews ausgesetzt - durch Politikberater (Auftragnehmer) wie politische Akteure (Auftraggeber). Die Namen der Interviewten tauchen zwar auf, aber es unterbleibt eine Zuordnung der jeweiligen Aussagen. Wieso Anonymität notwendig war, erschließt sich dem Rezensenten nicht. Zu Recht wird schließlich das allgemeine „Bestreben nach Transparenz“ (S. 221, S. 235) hervorgehoben. Über die Notwendigkeit eines solchen Kriterienkatalogs bestand bei den Befragten weithin Einigkeit, ebenso über die Notwendigkeit zu Sanktionierungen für jene, die sich nicht daran halten. Die Ergebnisse aus den Interviews hätten etwas klarer dargestellt werden können.

Leider ist das Deutsch des Verfassers recht gestelzt, fällt die Zahl der (sprachlichen wie inhaltlichen) Redundanzen beträchtlich aus. Und es bleibt zu hoffen, dass er bei eigener Beratung weniger kompliziert und umständlich formuliert. Die blutleere Form der Präsentation verhindert wohl eine größere Resonanz der Erkenntnisse Rafats. Das ist bedauerlich, da der Autor zumal im zweiten Teil seiner Untersuchung mit seinem Kriterienkatalog Neuland für die Forschung zur Politikberatung betritt.

Eckhard Jesse

Technische Universität Chemnitz eckhard.jesse@phil.tu-chemnitz.de 